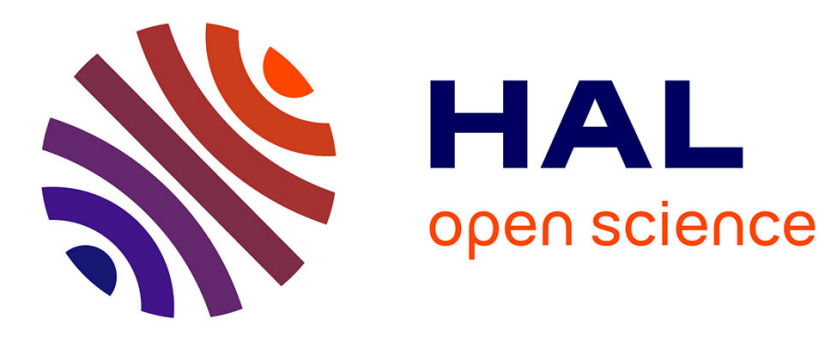

\title{
French guidelines on stereoelectroencephalography (SEEG)
}

Jean Isnard, Delphine Taussig, Fabrice Bartolomei, Pierre Bourdillon, Hélène

Catenoix, Francine Chassoux, Mathilde Chipaux, Stéphane Clemenceau, Sophie Colnat-Coulbois, Marie Denuelle, et al.

\section{To cite this version:}

Jean Isnard, Delphine Taussig, Fabrice Bartolomei, Pierre Bourdillon, Hélène Catenoix, et al.. French guidelines on stereoelectroencephalography $(\mathrm{SEEG})$. Neurophysiologie Clinique $=$ Clinical Neurophysiology, 2018, 48 (1), pp.5-13. 10.1016/j.neucli.2017.11.005 . hal-01744390v2

\section{HAL Id: hal-01744390}

\section{https://hal-univ-rennes1.archives-ouvertes.fr/hal-01744390v2}

Submitted on 15 Jun 2018

HAL is a multi-disciplinary open access archive for the deposit and dissemination of scientific research documents, whether they are published or not. The documents may come from teaching and research institutions in France or abroad, or from public or private research centers.
L'archive ouverte pluridisciplinaire HAL, est destinée au dépôt et à la diffusion de documents scientifiques de niveau recherche, publiés ou non, émanant des établissements d'enseignement et de recherche français ou étrangers, des laboratoires publics ou privés. 


\section{REVIEW ARTICLE}

\section{French Guidelines on Stereoelectroencephalography (SEEG)}

Jean Isnard ${ }^{\mathrm{a}, \mathrm{b}, *}$, Delphine Taussig ${ }^{\mathrm{c}}$, Fabrice Bartolomei ${ }^{\mathrm{d}, \mathrm{e}}$, Pierre Bourdillon ${ }^{\mathrm{a}, \mathrm{f,g}, \mathrm{h}}$, Hélène Catenoix $^{\mathrm{a}, \mathrm{b}, \mathrm{f}}$, Francine Chassoux ${ }^{\mathrm{i}}$, Mathilde Chipaux ${ }^{\mathrm{d}}$, Stéphane Clémenceau ${ }^{\mathrm{j}}$, Sophie ColnatCoulbois $^{\mathrm{k}}$, Marie Denuelle, Stéphane Derrey ${ }^{\mathrm{m}, \mathrm{n}}$, Bertrand Devaux ${ }^{\mathrm{i}}$, Georg Dorfmüller ${ }^{\mathrm{c}}$, Vianney Gilard ${ }^{\mathrm{m}, \mathrm{o}}$, Marc Guenot ${ }^{\mathrm{a}, \mathrm{f}}$, Anne-Sophie Job-Chapron ${ }^{\mathrm{p}, \mathrm{q}, \mathrm{r}}$, Elisabeth Landré ${ }^{\mathrm{i}}$, Axel Lebas $^{\mathrm{s}}$, Louis Maillard ${ }^{\mathrm{t}, \mathrm{u}}$, Aileen McGonigal ${ }^{\mathrm{d}, \mathrm{e}}$, Lorella Minottip ${ }^{\mathrm{p}, \mathrm{r}}$, Alexandra Montavont ${ }^{\mathrm{v}, \mathrm{a}, \mathrm{b}}$, Vincent Navarro $^{\mathrm{w}, \mathrm{x}, \mathrm{y}}$, Anca Nica $^{\mathrm{z}, \mathrm{aa}}$, Nicolas Reyns ${ }^{\mathrm{ab}, \mathrm{ac}}$, Julia Scholly ${ }^{\mathrm{ad}}$, Jean-Christophe Sol ${ }^{\mathrm{ae}}$, William Szurhaj ${ }^{\text {af,ag,ah }}$, Agnès Trebuchon ${ }^{\mathrm{d}, \mathrm{e}}$, Louise Tyvaert ${ }^{\mathrm{t}, \mathrm{u}}$, Maria Paola Valenti-Hirsch ${ }^{\text {ad }}$, Luc Valton ${ }^{\text {lai,aj }}$, Jean-Pierre Vignal ${ }^{\mathrm{t}, \mathrm{u}}$, Paul Sauleau ${ }^{\mathrm{ak}, \mathrm{al}}$

a. Hospices Civils de Lyon, Hospital for Neurology and Neurosurgery Pierre Wertheimer, Department of Functional Neurology and Epileptology, 59, boulevard Pinel, 69677, Broncedex, France

b. TIGER: Neuroscience research center of Lyon, INSERM U1028, CNRS 5292, Lyon, France

c. Paediatric Neurosurgery, Rothschild Foundation Hospital, 25-29, rue Manin, 75019 Paris, France

d. Aix Marseille University, Institut de Neurosciences des Systèmes, Marseille, F-13005, France

e. AP-HM, Hôpital de la Timone, Service de Neurophysiologie Clinique, Marseille, F-13005, France

f. Université de Lyon, Université Claude Bernard, Lyon, France

g. Brain and Spine Institute, INSERM U1127, CNRS 7225, Paris, France

h. Sorbonne Universités, Université Pierre et Marie Curie, Paris, France

i. Department of Neurosurgery, Unit of Epileptology, Sainte-Anne Hospital, 75014 Paris, France

j. Department of Neurosurgery, Groupe Hospitalier Universitaire de La Pitié-Salpêtrière, 47-83, Boulevard de l'Hôpital, 75013, Paris, France

k. Service de Neurochirurgie, Hôpital Central - CHU de Nancy, 29 Avenue du Maréchal de Lattre de Tassigny, 54000 Nancy, France

1. Explorations neurophysiologiques, Hôpital Purpan, Université de Toulouse, France

m. Department of Neurosurgery, Rouen University Hospital, Rue de Germont, Rouen, France.

n. INSERM 1073, Faculty of Medicine, Rouen, France

o. Microvascular Endothelium and Neonate Brain Lesions Laboratory, INSERM ERI 28, Faculty of Medicine, Rouen, France

p. Neurology Department, CHU Grenoble Alpes, Grenoble, France

q. University Grenoble, France 
r. Grenoble Institute of Neuroscience (GIN), INSERM, U1216, Grenoble, France.

s. Department of Neurophysiology, , Rouen University Hospital, Rue de Germont, Rouen, France.

t. Neurology Department, CHU de Nancy, France

u. CRAN UMR 7039 CNRS Lorraine-Université, France

v. Service Épilepsie, Sommeil et Explorations Fonctionnelles Neuropédiatriques, HFME, 59, boulevard Pinel, 69677Bron cedex, France

w. AP-HP, GH Pitié-Salpêtrière-Charles Foix, EEG and Epilepsy Unit, F-75013, Paris, France.

x. Centre de référence des Epilepsies rares

y. Brain and Spine Institute (ICM; INSERM, UMRS 1127; CNRS, UMR 7225), Paris, France.

z. Service de Neurologie, CHU de Rennes, 2, rue Henri-le-Guilloux, 35033 Rennes, Cedex 9, France

aa. INSERM UMR 1099, Rennes2, France

ab. Department of Neurosurgery, University Hospital, Lille, France.

ac. Univ. Lille, INSERM, CHU Lille, U1189-ONCO-THAI-Image Assisted Laser Therapy for Oncology, F-59000, Lille, France.

ad. Medical and Surgical Epilepsy Unit, Hautepierre Hospital, University of Strasbourg, France

ae. Service de neurochirurgie, CHU de Toulouse, université Paul-Sabatier, 31059 Toulouse, France.

af. Department of clinical neurophysiology, Lille University Hospital, France

ag. INSERM U1171, University of Lille, 59000 Lille, France

ah. Faculté de Médecine Henri-Warembourg, 59045 Lille Cedex, France

ai. Université de Toulouse, France

aj. CerCo, Centre de recherche Cerveau et Cognition UMR 5549 - CNRS, Toulouse, France.

ak. Unité des Explorations Fonctionnelles Neurologiques, CHU de Rennes, 2, rue Henri-le-Guilloux, 35033 Rennes Cedex 9, France

al. EA 4712 « Comportement et Noyaux Gris Centraux », Université de Rennes 1, Faculté de Médecine, avenue Léon-Bernard, 35043 Rennes, France

Corresponding author: Jean Isnard, Service de Neurophysiologie et d'Épileptologie, Hôpital Neurologique P. Wertheimer, Hospices Civils de Lyon, 59 boulevard Pinel, 69677 Bron cedex, France. E-mail address:jean.isnard@chu-lyon.fr. 


\section{Summary}

Stereoelectroencephalography (SEEG) was designed and developed in the 1960s in France by J. Talairach and J. Bancaud. It is an invasive method of exploration for drug-resistant focal epilepsies, offering the advantage of a tridimensional and temporally precise study of the epileptic discharge. It allows anatomo-electrical correlations and tailored surgeries. Whereas this method has been used for decades by experts in a limited number of European centers, the last ten years have seen increasing worldwide spread of its use. Moreover in current practice, SEEG is not only a diagnostic tool but also offers a therapeutic option, i.e., thermocoagulation. In order to propose formal guidelines for best clinical practice in SEEG, a working party was formed, composed of experts from every French centre with a large SEEG experience (those performing more than 10 SEEG per year over at least a 5 year period). This group formulated recommendations, which were graded by all participants according to established methodology. The first part of this article summarizes these within the following topics: indications and limits of SEEG; planning and management of SEEG; surgical technique; electrophysiological technical procedures; interpretation of SEEG recordings; and SEEG-guided radio frequency thermocoagulation. In the second part, those different aspects are discussed in more detail by subgroups of experts, based on existing literature and their own experience. The aim of this work is to present a consensual French approach to SEEG, which could be used as a basic document for centers using this method, particularly those who are beginning SEEG practice. These guidelines are supported by the French Clinical Neurophysiology Society and the French chapter of the International League Against Epilepsy.

\section{Keywords}

adults; children; drug-resistant epilepsy; epilepsy surgery; focal epilepsy; guidelines; invasive exploration; stereo-electroencephalogram; thermocoagulations 


\section{Overview}

The stereoelectroencephalography (SEEG) method was invented over 50 years ago by Jean Bancaud and Jean Talairach at the Sainte Anne Hospital in Paris. This method is based on an integrated system allowing the placement of electrodes within the brain substance via a transcranial route. It thus became possible to record ictal electrical activity within predefined cortical targets in order to perform subsequent ablation. The innovative idea at the origin of this theory is that the semiology of focal seizures carries crucial information with regards to the neural origins of the seizure, and that by rigorous analysis it is possible to identifier the regions in which epileptic discharges arise. The integration of clinical observation with the analysis of peri-ictal EEG data allows anatomical-electroclinical correlations to be established. These correlations form the basis of hypotheses regarding the topographical characteristics of the epileptic network, which the goal of the SEEG exploration is to confirm or refute.

In this context, the electrode implantation is tailored according to each individual case: every implanted electrode represents a specific question to be answered. The recorded electrical signal will thus provide responses only to these questions. If the hypotheses are poorly formulated, or irrelevant, rendering the placement of electrodes suboptimal, the "answers" provided by the SEEG will be unintelligible, or worse, erroneous with a subsequent risk of surgical failure. Clearly, it is therefore not the quality of recorded signal nor the spatio-temporal resolution of the SEEG data that make the method so original, but rather the process of reflection prior to implantation, in which the three-dimensional organization of the epileptic network is envisaged and the implantation scheme planned in such a way as to allow this to be objectified. This constitutes a fundamental principle of the SEEG: a clear hypothesis must precede the implantation.

This principle being well established, the SEEG has evolved considerably since the time of its creation. On one hand, technical progress has allowed more ambitious exploration schemes to be proposed, in particular because strict orthogonality of implanted electrodes is no longer mandatory, and oblique trajectories can be used to reach less accessible cortical structures (such as the insula). On the other hand, the various non-invasive modalities that have been developed over the last 20 years, such as positron emission tomography (PET), magnetoencephalography (MEG), subtraction ictal single-photon emission computed tomography (SPECT) coregistered to MRI (SISCOM), high resolution EEG and so on, provide important data that can be integrated for each patient. These techniques have a crucial place alongside the anatomical electroclinical analysis, in particular during the formulation of hypotheses. Such progress has indeed allowed the clinical indications for stereotaxic explorations to be directed towards more complex cases, and inversely to eliminate the need for SEEG in some other patients who would previously have been candidates (for example, epilepsies related to tumors and/or involving the temporal lobe) but whom nowadays can be operated following non-invasive recording. Finally, more recently, a new therapeutic and diagnostic aspect of the SEEG exploration has emerged, through the 
possibility of performing thermocoagulation in order to produce multiple targeted lesions within the SEEG-defined epileptogenic network directly via the implanted electrodes.

Over a period of many years, the above-mentioned evolution and development of SEEG took place within the strict framework established by the fathers of the SEEG method, who formalized this in a first article of recommendations [10]. In fact, prior to 1990, the practice of SEEG was essentially limited to the Sainte-Anne hospital in Paris. Its use then spread within France (Rennes, Marseille, Grenoble) and in Italy (Milan), through the influence and under the guidance of the two main pupils of Jean Bancaud: Patrick Chauvel and Claudio Munari. The former in particular was a key figure in training new teams in epilepsy surgery through an annual training programme of theoretical and practical SEEG teaching in France. This major educational initiative has borne its fruit and has allowed development of the practice of SEEG in France that remains faithful to the spirit of its founding fathers, while at the same time incorporating new technological developments that allow evolution of the method as described above. A survey performed at the beginning of this work, including all 15 French centers regularly performing SEEG (minimum 10 explorations/year), showed that the average number of patients undergoing subsequent resective surgery for epilepsy increased from 20 to 223 per year between 1990 and 2017. Such expansion goes hand in hand with increasing technical expertise, allowing most centers to perform SEEG in difficult cases, with $48-87 \%$ of patients subsequently undergoing epilepsy surgery following SEEG exploration. In parallel, the acceptability of SEEG exploration has also improved, through better staffing of epilepsy monitoring units throughout the recording period (24-hour monitoring under the surveillance of specialized, dedicated nursing staff being available in 12/15 French centers). This has also allowed reduction of the average recording duration to 8.5 days. The virtual disappearance of the practice of pre-operative scalp hair removal before SEEG has evolved over the same period: only 2 of 15 centers continue to employ this practice, with an average number of implanted electrodes of 10-13 per center (range 5-23). However, more recently the success and the diffusion of the SEEG method have expanded exponentially, which creates the risk of deviation from its fundamental principles. In the last 5 years in particular, there has been a huge leap in international interest in SEEG and the traditional method of training by apprenticeship is no longer a guaranteed means of transmitting the mandatory theoretical and practical knowledge and experience. There is indeed a significant risk of losing sight of the theoretical framework of SEEG and reducing the practice to a mere technical procedure destined to consolidate the results of non-invasive investigations. Thus, the recommendations proposed here are designed not only to provide practical guidelines but to reinforce the importance of the theoretical framework of SEEG, which is the core heritage transmitted from its creators. The visionary SEEG method has provided us with a unique means of understanding the epileptic condition; its purpose and meaning must be perpetuated and developed, and not sacrificed by misjudged, anarchical development. 


\section{Methodology}

The overall methodology was based on the methodology used to produce the French recommendations on electroencephalography [2,3]. However, this had to be adapted for the present work because of the specificity of SEEG. Experts with an experience of more than 10 SEEG a year for more than 5 years participated in this publication. As the number of French experts is limited, it was decided that all should be authors. A plenary meeting decided the topics to be dealt with and 6 subgroups made up of 4 to 6 experts were constituted. By consensus agreement, issues specific to pediatric SEEG will be dealt with within each topic, rather than proposing a separate set of guidelines. Each group proposed recommendations on its topic, which were subseuqently discussed and graded by the complete workgroup. Each participant graded each guideline according to the RAND/UCLA method [4]. They graded the items within their field of expertise from 1 to 9 (1 corresponding to "complete disagreement" or "total absence of evidence" or " absolute contraindication" and 9 corresponding to “complete agreement" or "validated evidence" or "'absolute indication"). Three areas were defined according to the position of the median score: the $(1-3)$ area corresponded to the "disagreement" area; the (4-6) area to the "unresolved", area; and the (7-9) area to the "agreement" area. The agreement, disagreement or unresolved status was deemed "strong" if the median interval was located within one of these three areas $(1-3),(4-6)$ or $(7-9)$. The agreement, disagreement or unresolved status was deemed "weak" if the median interval overlapped a grading area (e.g. interval [1-4] or interval [6-8]). Authors had the possibility of not grading an item if they felt that their opinion was not relevant for a specific question. After four grading rounds and the possibility of discarding a maximum of one deviating grade, the workgroup wrote consensual guidelines for most items addressed. In case of lack of consensus, the mention "non-consensual agreement" was added and the reader was invited to look at the detailed chapter for more information on this item. The first part of the publication is made up of these guidelines.

The second part is made up of the 6 articles that address in detail the 6 topics for which guidelines were graded. Each was written by the expert subgroup who prepared the proposals for the guidelines. In each subgroup, the literature search focused on medical articles in the French or English language (PubMed, Web of Sciences, up to June 2017, using different keywords for each chapter). Authors were required to discuss the items for which no consensus was reached. In the past years, several reviews of SEEG have been published, in particular the invited reviews in Journal of Clinical Neurophysiology (2016) $[3,4,6,7,11]$. However, the specificity of the present publication is in its representing the consensual work of the complete group of SEEG experts in France. 


\section{Overview}

As mentioned above, the plan of this document was developed during plenary meetings of the workgroup. The workgroup decided to categorize the recommendations into 6 themes:

- Indications and limits of stereoelectroencephalography (SEEG)

- Planning and management of SEEG

- Surgical technique

- Electrophysiological technical procedures

- Interpretation of SEEG recordings

- SEEG-guided radiofrequency thermocoagulation

\section{SEEG recommendations}

\section{I) Indications and limits of stereoelectroencephalography (SEEG)}

- SEEG is indicated when a surgical hypothesis exists, but when anatomo-electroclinical data collected during the non-invasive phase are insufficiently concordant regarding the supposed localization of the region responsible for seizures (epileptogenic zone, EZ) and/or its relationship with functional areas

- SEEG is indicated to confirm a main hypothesis and to eliminate alternative hypotheses of the localization of the EZ.

- SEEG is also indicated to establish a surgical strategy.

- SEEG can be proposed for the purpose of thermocoagulations.

- SEEG is the most appropriate method for exploring: 1 / sulcal cortical zones including focal cortical dysplasia, 2/ deeply located cortical structures (for instance the insulo-opercular system, the limbic system), and 3/ deeply located or periventricular lesions (for instance periventricular heterotopia and hypothalamic hamartoma).

- In epilepsies with early involvement of motor or speech areas, arguments in favor of functional reorganization must be sought before considering that SEEG is indicated (non-consensual agreement; see the detailed chapter "Indications and limits of SEEG").

- SEEG is preferred to subdural explorations in the majority of cases because of its lower morbidity and better tolerance.

- SEEG is preferred to subdural exploration when bilateral.

- SEEG is preferred to subdural exploration when the patient has previously undergone a cerebral surgical intervention with craniotomy.

- SEEG is preferred to subdural explorations in cases of MRI-negative epilepsies.

- The main limitation of SEEG is the sampling bias. 
- Another limitation of SEEG is the difficulty to precisely localize functional areas, in particular language areas.

- In temporal lobe epilepsies with mesial symptomatology or lesion, SEEG is indicated when the extra-limbic or extra-temporal cortex, or the contralateral temporal lobe seem to show early involvement when considering the non-invasive data.

- In patients with temporal lobe epilepsy, the lack of radiological features of hippocampal sclerosis constitutes a strong argument in favor of performing SEEG prior to surgical treatment (non-consensual agreement, see the detailed chapter "Indications and limits of SEEG").

- In temporal lobe epilepsies, when Wernicke's area seems to be involved early in the course of seizures, invasive exploration is mandatory. The choice between SEEG and subdural exploration must be discussed (non-consensual agreement, see the detailed chapter "Indications and limits of SEEG").

- In epilepsies with temporal semiology, SEEG is indicated when extemporal origin or extension, or a bilateral epileptogenic zone are suggested by non-invasive explorations.

- In lesional extra-temporal epilepsies, SEEG can be indicated to define the border of the epileptogenic network and its relationship with functional areas.

- In lesional extra-temporal epilepsies, SEEG can be indicated in cases of discrepancy between the topography of the lesion and the electroclinical semiology.

- In lesional extra-temporal epilepsies, the indication of SEEG depends on the nature of the lesion.

- In hypothalamic hamartoma-associated epilepsies, SEEG is indicated only when an EZ independent of the hamartoma is suspected.

- In epilepsies associated with multiple lesions (tuberous sclerosis, focal cortical dysplasia, nodular periventricular heterotopia, cavernous angioma), SEEG is indicated when non-invasive data provide strong evidence that the $\mathrm{EZ}$ is unique or is largely predominant.

- In unilateral polymicrogyria (PMG)-associated epilepsies, SEEG is the method of choice for determining the involvement of the PMG in the organization of the EZ.

- $\quad$ SEEG can be proposed in bilateral PMG if non-invasive data suggest a unilateral EZ.

- In childhood epilepsies, SEEG can be proposed when there is a hypothesis about the localization of the EZ, taking into account the difficulties in anatomo-electroclinical correlations.

- Some age-related encephalopathies, in particular infantile spasms and electrical status epilepticus during sleep, can be indications for SEEG when electroclinical and/or imaging data favour a focal origin. 


\section{II) Planning and management of SEEG}

- Planning of stereoelectroencephalography (SEEG) must meet the following objectives: 1) to define the epileptogenic zone (EZ), 2) to study its relationship with functional areas and 3) to evaluate the possibility of surgical resection.

- SEEG planning is based on the integration of presurgical assessment data which must be as comprehensive as possible and depends on video-EEG seizure recordings (including all seizure types), structural and functional imaging according to specific protocols.

- The implantation scheme is planned by the multi-disciplinary medical-surgical team.

- It is necessary to elaborate a main hypothesis on which to focus the exploration; alternative hypotheses are necessary in order to avoid sampling error.

- The number of electrodes required is not absolutely fixed but, apart from special cases (SEEG complement or planned thermocoagulation), when less than 6 electrodes are considered the relevance of the SEEG should be questioned, and above 15 electrodes, it is necessary to consider further investigations that could reduce the number of planned electrodes (nonconsensual agreement, see the detailed chapter "Planning and management of SEEG").

- Determination of hemispheric dominance for language before implantation is appropriate, if evaluable according to the age and the cooperation of the patient (non-consensual agreement, see the detailed chapter "Planning and management of SEEG").

- One hemisphere should be preferentially explored, but if when contralateral electrodes are indicated, these should be placed symmetrically if possible. On the other hand, bilateral and symmetrical exploration, with the same number of electrodes in both hemispheres, is not recommended.

- The placement of electrodes in eloquent areas (language, motor cortex) must be avoided unless the problem is precisely the question of their involvement.

- In temporal lobe epilepsy, sampling usually involves the mesial temporal structures (hippocampus and amygdala), the entorhinal cortex, the middle temporal gyrus and basal cortex, the superior temporal gyrus, the temporal pole and the insular cortex (floor).

- In temporal lobe epilepsy involving extratemporal or contralateral structures, other regions can be sampled (orbito-frontal cortex, perisylvian region, temporoparietal junction, contralateral mesial temporal structures).

- In frontal lobe epilepsy, before discussing SEEG planning, a preliminary robust hypothesis in terms of lateralization and localization (anterior versus posterior, mesial versus dorso-lateral) is mandatory.

- In frontal lobe epilepsy, the various structures involved in an anatomo-functional network should be explored (orbital cortex, anterior cingulate gyrus, insula in anterior frontal epilepsy 
for example; supplementary motor area, cingulate gyrus, motor cortex in cases of posterior frontal epilepsy).

- To sample the cortical convexity, the choice of oblique trajectories may be useful.

- Sampling of the central region must take into account the particular risks in case of hemorrhagic complication and the risk-benefit ratio of each electrode must be specifically evaluated.

- Posterior epilepsies most often require multilobar sampling, unilateral or bilateral, with particular concern for the pathways of propagation and the involvement of functional structures.

- In perisylvian epilepsies, it is necessary to explore the insula and the opercula.

- Exploration of the insula can be performed obliquely or orthogonally, the technique of implantation and the choice of the trajectories depending on the experience of the teams and the vascular constraints.

- In lesional epilepsies, sampling depends on the type of causal lesion but requires the placement of one or several intralesional electrodes (except in the case of vascular malformation or cystic lesion) and the placement of perilesional electrodes.

- The SEEG is conducted after evaluation of the risk-benefit ratio, by a medical and paramedical team specifically trained in this methodology.

- Information given to the patient, and to legal guardians if the patient is minor or under guardianship, must be written in the patient's chart.

- Continuous SEEG-video monitoring by dedicated staff is preferable.

- Close monitoring of the patient by the medical and paramedical team in a dedicated environment is mandatory.

- Night recordings are informative and suitable whenever monitoring and safety conditions are met.

- Daytime sleep recordings are sometimes useful for recording seizures related to sleep or waking.

- The presence of a relative may be sought in some patients to increase safety and facilitate the progress of the exploration; this is required if there is a risk of agitation, and in children.

- SEEG is usually carried out under progressive antiepileptic drug withdrawal except in the case of frequent (at least daily) seizures.

- Recording at least one spontaneous seizure reproducing the known semiology is recommended; recording several seizures is useful to verify their homogeneity especially if the patient has several types of seizures.

- In focal cortical dysplasia, subclinical seizures and habitual seizures obtained after lowfrequency stimulation have the same value as spontaneous seizures (non-consensual agreement, see the detailed chapter "Planning and management of SEEG"). 
- In hippocampal structures, a habitual seizure reproduced after low frequency stimulation has similar value of that of spontaneous seizures (non-consensual agreement, see the detailed chapter "Planning and management of SEEG").

- Secondary appearance of permanent slow waves or deterioration of the background activity during SEEG suggest a hematoma and indicate the need for emergency CT scan.

- Duration of SEEG should be limited to the time required to obtain relevant information on the organization of the EZ and to propose a surgical procedure.

- Hands-on clinical training of medical practitioners is recommended and should occur in collaboration with experienced teams (minimum 5 years of experience), with active participation in all stages of SEEG including interpretation (duration of several months and / or at least 10 SEEG). EEG nurses and technicians must also receive specific training.

\section{III) Surgical techniques}

- It is strictly necessary to deliver clear, honest, and appropriate information to the patient.

- SEEG is performed under general anesthesia.

- Pre-operative biological investigation should include hemostasis investigation. Results of these investigations must be discussed together with hematologists, if abnormalities are detected.

- Prophylactic antibiotic treatment must be given, according to the current protocol in use in the center.

- It is unnecessary to crop the patient's hair; the literature does not provide any data in favour of this.

- It is necessary to perform vascular imaging to minimize the peri-operative bleeding risk.

- Vascular imaging can be performed using digital cerebral angiography, angio-MRI scan, CTangiography or contrast-enhanced MR-scan.

- Vascular imaging can be performed some days before, or on the day of, implantation.

- MRI is the reference morphological imaging procedure, used for targeting and trajectory calculations.

- Morphological MRI scan can be performed some days before, or on the day of, implantation.

- Morphological imaging data must be coregistered with vascular imaging data.

- Targeting and trajectory calculation are carried out by means of any suitable software available in the center.

- It is necessary to use specific, dedicated, surgical instruments, adapted to the devices being implanted.

- The penetration sites of the electrodes must not be at risk of entering any air sinus.

- The use of intra-operative imaging data can be useful, but is not compulsory. 
- Once implanted, each electrode must be clearly externally identified, and protected by means of a firm head bandage.

- Careful clinical monitoring of awakening after anesthesia and of the immediate post-operative neurological status must be performed.

- If any new neurological deficit is noticed, neuroimaging must immediately be performed, consisting at least of a CT scan and, if possible an MRI scan, in order to be able to immediately reoperate the patient if necessary (e.g. in the case of intracranial hematoma).

- Post-operative admission in intensive care unit is not compulsory in the absence of complication (non-consensual agreement, see the detailed chapter 'Surgical techniques').

- As soon as the patient is awake with normal neurological status, he or she can be transferred to the neurosurgery department, or directly to the epilepsy monitoring unit (non-consensual agreement, see the detailed chapter 'Surgical techniques').

- It is recommended to perform early (within 24 hours) post-operative imaging (MR-scan alone, or CT-scan coregistered to MR-scan), to be sure of the absence of any asymptomatic complication, and to check the exact position of each implanted electrode.

- Electrode removal can be carried out at the patient's bedside or in the operating room (nonconsensual agreement, see the detailed chapter "Surgical techniques").

- In young pediatric patients, it is recommended to perform electrode removal under local, or brief general, anesthesia, and to use sutures (non-consensual agreement, see the detailed chapter "Surgical techniques").

- It is strongly recommended to ensure that every removed electrode is intact. If not, skull X-rays must be immediately performed.

- It is recommended to keep the patient in the hospital for at least one day after electrode removal.

- It is accepted that the minimal acceptable skull bone thickness to perform SEEG is $2 \mathrm{~mm}$.

- The literature does not provide any data in favor of the need to perform neuroimaging following electrode removal (non-consensual agreement, see the detailed chapter "Surgical techniques").

\section{IV) Electrophysiological technical procedures}

- After implantation of the electrodes in the operating room, SEEG recording is begun as soon as possible.

- Brain CT or MRI should be performed with the electrodes in place.

- The acquisition system must have at least 128 channels and ideally 256 channels.

- Care should be taken not to record with a $50 \mathrm{~Hz}$ filter, to avoid misinterpretation of sector artefacts that may make it difficult to detect low-voltage rapid discharges. 
- When the number of contacts is greater than the number of available recording channels, the activity of each contact will be visualized before any selection of the contacts of interest. The contacts will be selected according to their site in the brain, with exclusion where necessary of contacts located in the white matter (non-consensual agreement, see the detailed chapter "Electrophysiological technical procedures").

- For the summary montage, the contacts exploring the white matter or located outside the brain will preferably be discarded.

- When the ground and the reference contacts are distinct, the reference will be chosen from the recording contacts located in the bone, the white matter or an accessible location on the scalp (between $\mathrm{Cz}$ and $\mathrm{Fz}$ ).

- The ground contact will be located in the bone, in the white matter or an accessible cutaneous site (earlobe or extracephalic).

- In children, the reference contact and the ground contact will preferentially be contacts located in the white matter.

- Simultaneous and synchronous recording of scalp EEG is not necessary but can be performed by means of a few electrodes.

- Simultaneous and synchronous recording of EMG polygraphy is useful when exploring motor semiology.

- Simultaneous sleep polygraphic recording (scalp, EOG and EMG) is useful for assessing the evolution of cortical activity during sleep.

- The recording of electrocardiography and pulse oximetry are necessary.

- Synchronized video during the recording of intracerebral activity is essential for electroclinical correlations.

- The video equipment must allow analysis of detailed and global seizure semiology.

- For night recordings, video equipment must include an infrared camera.

- The sampling frequency must be at least $256 \mathrm{~Hz}$ and ideally $512 \mathrm{~Hz}$ for a standard acquisition.

- Hyperventilation (3-6 minutes) should be used to increase likelihood of occurrence of interictal abnormalities and seizures.

- Intermittent photic stimulation (IPS) is useful in the exploration of posterior cortex epilepsies.

- The provocation of seizures by individual factors reported by the patient is used, for example partial sleep deprivation, surprise or sudden noises in cases of startle epilepsy.

- Intracerebral electrical stimulation has two main aims: to reproduce ictal clinical expression and to perform functional mapping of the regions explored. The stimulation data are used to study inter-structural functional connectivity.

- Electrical stimulations are usually performed after recorded spontaneous seizures. 
- Electrical stimulations are performed between two contiguous contacts of the electrode using bipolar and biphasic current.

- The usual parameters for shock stimulation (low frequency) are: (1) $1 \mathrm{~Hz}$ frequency, (2) shock duration of 0.5 to 3 milliseconds, (3) intensity of 0.5 to 4 milliamps, (4) stimulation duration of 20 to 60 seconds.

- The usual parameters for train stimulation (high frequency) are: (1) $50 \mathrm{~Hz}$ frequency, (2) shock duration of 0.5 to 1 milliseconds, (3) intensity of 0.5 to 5 milliamps, (4) stimulation duration of 3 to 8 seconds.

- Shock stimulations are useful for triggering seizures, especially in the hippocampus and in focal cortical dysplasias, for functional mapping in particular of primary areas, and for study of functional connectivity.

- Train stimulations are useful for triggering seizures and for performing functional mapping.

- Train stimulations should be used with caution in the central region because of risk of triggering generalized tonic-clonic seizures; only short and low-intensity trains should be employed.

- It is important to wait between stimulations for electrophysiological activity to return to its baseline level.

- Functional mapping can be supplemented by the recording of evoked activities (evoked potentials or oscillatory activity) in response to certain stimuli.

- Physiological potentials and reactivity of cerebral rhythms can be objectified directly using stimuli such as sudden noises, motor maneuvers and IPS.

- In uncooperative children, localization of the motor areas can be performed during sleep, using train stimulations.

\section{V) Interpretation of SEEG recordings}

- SEEG interpretation must be carried out or supervised by an experienced epileptologist/neurophysiologist trained in the field.

- Visual analysis of SEEG traces will use referential and bipolar montages in a global montage, or more selected channels.

- Interpretation must take into account the different explored brain regions that may disclose different SEEG physiological patterns. This activity may vary according to patients' activities and state of alertness.

- Therefore, precise knowledge of the anatomical location of electrode contacts is crucial for interpretation. 
- Interictal activity is analyzed at rest and in different states of alertness, and also after various activation procedures (intermittent photic stimulation, hyperventilation, movement, eye closure, etc.).

- An important step is analysis of interictal spikes (IS). Their anatomical distribution (classically defining the irritative zone), and their frequency in each region must be studied. Background activity must be analyzed in each explored brain region, particularly looking for slowing or other alteration.

- Morphological characteristics of IS (sharpness, duration, amplitude, association with fast activities, etc.) must be analyzed.

- Subclinical rhythmic discharges are important to analyze, since they may help to define the epileptogenic zone (EZ).

- Ictal discharges are analyzed in parallel with interictal activity.

- Seizures are the main object of study of the SEEG, via the process of anatomical- electroclinical correlations.

- The first SEEG ictal modifications are often characterized by rapid discharge (low voltage fast activities) and generally define the SEEG seizure onset zone.

- Spontaneous seizures and seizures provoked by electrical stimulation are analyzed by studying electrical changes and the corresponding clinical modifications (peri- and post-ictal).

- The EZ is characterized by a certain dynamic that must be described: for example, preictal changes (spike, slow waves, etc.) followed by a rapid discharge; however, other kinds of patterns of seizure onset are possible.

- Localized electrical depression or slowing during the post-ictal period may have a good localizing value.

- The spatial extension of the EZ must be defined: involved contacts, brain regions involved at the seizure onset and during early spread.

- Clinical semiology must be interpreted in the light of the electrical changes. The first electrical changes must precede the clinical signs and/or symptoms.

- Analysis of semiology is based on the study of ictal and postictal clinical expression.

- Electrical stimulations must be interpreted in terms of functional responses (for functional mapping) and require analysis of specific protocols (for language testing, motor function, visual function, etc.)

- These functional responses must be compared to other modalities such as evoked potentials and fMRI.

- The intensity of stimulation and the associated electroclinical responses (with or without afterdischarges) allows evaluation of the tissue's excitability. 
- Seizures triggered by stimulation have an important diagnostic value when they reproduce (at least in part) the usual clinical pattern of the patient's seizures.

- It should however be noted that stimulation of regions distant from the EZ (but connected to the EZ) may induce seizures.

- The absence of seizures triggered by stimulation has no prognostic value for defining the EZ but may also depend on epilepsy etiology.

- Quantification and analysis of signal is recommended to help the interpretation (non-consensual agreement, see the detailed chapter 'Interpretation of SEEG recordings').

- Signal analysis may include quantification of spiking activity in different states (waking, sleep, preictal, postictal).

- Analysis of ictal onset can be facilitated by methods like the epileptogenicity index (EI) or gamma activity map.

- SEEG interpretation provides an anatomical and volumetric estimation of the EZ, based on SEEG data but also through integrating knowledge of the anatomical systems involved, and may also be helped by the non-invasive data.

- SEEG conclusions must provide an anatomical definition of brain regions engaged in the production of interictal and ictal activity, and their relationship with a possible lesion.

- Definitive conclusions (with a possible resection scheme) are presented during a multidisciplinary conference meeting.

\section{VI) SEEG-guided Radiofrequency Thermocoagulation}

- The main therapeutic indication of SEEG-guided radiofrequency thermocoagulations (SEEGguided RF-TC) is the presence of contraindication to surgical resection, because of morphological or functional anatomical definition of the epileptogenic zone (EZ).

- When phase I investigations do not lead to a surgical indication, SEEG can be performed with the purpose of performing SEEG-guided RF-TC.

- The patient should be informed about the possibility of SEEG-guided RF-TC, when available, prior to the SEEG.

- SEEG-guided RF-TC is not a contraindication to subsequent conventional surgery and has no influence on this.

- Conventional EZ surgery is better than SEEG-guided RF-TC when feasible.

- SEEG-guided RF-TC lesion is a thermocoagulation performed between two contiguous electrode contacts. The number of thermocoagulations is determined by the size of the targeted zone (non-consensual agreement, see the detailed chapter "Coagulations").

- The power of the delivered current should be gradually increased until this parameter, or the impedance, collapses. Duration must be less than a minute. 
- All thermocoagulations between two contiguous contacts should be preceded by a direct electric stimulation to confirm the safety of the target.

- Motor deficit occurring during direct electric stimulation is predictive of a similar impairment in the case of thermocoagulation on this target.

- Non-motor neurological impairment occurring during a direct electric stimulation of the target calls into question the benefit/risk ratio of the procedure, which must be evaluated in the context of each individual case.

- Motor neurological impairment occurring during a direct electric stimulation of the electrode contacts adjacent to the target also raises questions about the benefit/risk ratio of the procedure, and must again be evaluated in the context of each individual case.

- Any pain reported during thermocoagulation requires cessation of the procedure.

- When general anesthesia is not needed, clinical neurological monitoring has to be performed during SEEG-guided RF-TC.

- SEEG-guided RF-TC are usually performed at the end of the SEEG recording period.

- Continued SEEG recording can be useful after SEEG-guided RF-TC.

- Thermocoagulation targets are defined according to EZ markers, such as a typical low amplitude fast pattern and seizure triggering after direct electric stimulation (non-consensual agreement, see the detailed chapter 'SEEG-guided Radiofrequency Thermocoagulation').

- Thermocoagulation targets are not defined according to interictal activities alone, except for focal cortical dysplasia when a typical electrophysiological signature is present.

- Dipoles used for SEEG-guided RF-TC are defined by ictal activity. These can be located either in grey matter (EZ) or white matter (propagation pathways).

- The presumed ictal onset zone can be over-implanted during SEEG in order to maximize benefit from a potential SEEG-guided RF-TC (non-consensual agreement, see the detailed chapter "Coagulations").

- SEEG-guided RF-TC is an option for treating deep heterotopias when their involvement in the EZ is established.

- SEEG-guided RF-TC is an option for epilepsy related to hypothalamic hamartoma, when surgical resection/disconnection is not feasible or is deemed too risky (due to anatomic location and/or size of the hypothalamic hamartoma).

- Surgery, rather than SEEG-guided RF-TC, is the first-line therapeutic option in temporal lobe epilepsy because of higher and more durable efficacy.

- Multiple SEEG-guided RF-TC can be performed in cases of relapse following a previous procedure.

- Improvement of epilepsy, even partial, during at least the two months following SEEG-guided RF-TC is a positive predictive factor of good outcome after conventional surgery (non- 
consensual agreement, see the detailed chapter "SEEG-guided Radiofrequency

Thermocoagulation").

- SEEG-guided RF-TC may be an alternative to conventional surgery when the EZ has been proved to be very focal (non-consensual agreement, see the detailed chapter "Coagulations").

- SEEG-guided RF-TC should be performed without anesthesia to allow for neurological clinical monitoring during the procedure (non-consensual agreement, see the detailed chapter "SEEGguided Radiofrequency Thermocoagulation").

\section{Disclosure of interest}

The authors declare that they have no conflicts of interest concerning this article.

\section{Acknowledgments}

The authors would like to thank the Société de Neurophysiologie Clinique de Langue Française (SNCLF) and the Ligue Française Contre l'Epilepsie (LFCE) for their logistic and financial support for this work. They are very grateful to Aileen McGonigal for English editing.

\section{References}

1. André-Obadia N, Lamblin M-D, Sauleau P. French recommendations on electroencephalography. Neurophysiol Clin 2015; 45: 1-17.

2. André-Obadia N, Sauleau P, Cheliout-Heraut F, Convers P, Debs R, Eisermann M, et al. [French guidelines on electroencephalogram]. NeurophysiolClin 2014; 44: 515-612.

3. Bulacio J, Chauvel P, McGonigal A. Stereoelectroencephalography: Interpretation. J Clin Neurophysiol 2016; 33: 503-10.

4. Cardinale F, Casaceli G, Raneri F, Miller J, Lo Russo G. Implantation of stereoelectroencephalography electrodes: a systematic review. J Clin Neurophysiol 2016; 33: 490-502.

5. Fitch K, Bernstein S, Aguilar M, Burnand B, LaCalle J, Lazaro P, et al., The RAND/UCLA Appropriateness Method User's Manual, 2001.

6. Gonzalez-Martinez J. The stereo-electroencephalography: the epileptogenic zone. J Clin Neurophysiol 2016; 33: 522-9.

7. Kalamangalam G, Tandon N. Stereo-EEG implantation strategy. J Clin Neurophysiol 2016; 33: 483-9.

8. Lhatoo S, Lacuey N, Ryvlin P. Principles of Stereotactic Electroencephalography in Epilepsy Surgery. J Clin Neurophysiol 2016; 33: 478-82.

9. Schuele S. Stereoelectroencephalography. J Clin Neurophysiol 2016; 33: 477. 
10. Talairach J, Bancaud J, Szikla G, Bonis A, Geier S, Védrenne C. Approche nouvelle de la neurochirurgie de l'épilepsie. Méthodologie stéréotaxique et résultats thérapeutiques. Neurochirurgie 1974; 20(suppl 1): 1-249.

11. Trébuchon A, Chauvel P. Electrical Stimulation for Seizure Induction and Functional Mapping in Stereoelectroencephalography. J Clin Neurophysiol 2016; 33: 511-21. 